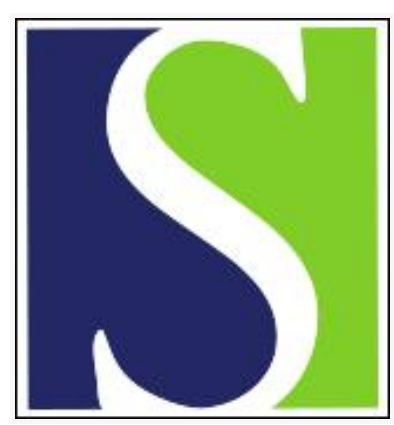

Scand J Work Environ Health 1997;23(1):5-14

https://doi.org/10.5271/sjweh.172

Issue date: Feb 1997

Need for a European approach to the effects of extremely low-frequency electromagnetic fields on cancer

by ELF-EMF European Feasibility Study Group

Key terms: brain tumor; breast cancer; childhood cancer; electromagnetic fields; leukemia

This article in PubMed: www.ncbi.nlm.nih.gov/pubmed/9098906

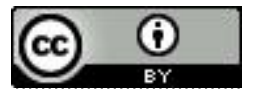




\title{
Need for a European approach to the effects of extremely low-frequency electromagnetic fields on cancer
}

\author{
by ELF-EMF European Feasibility Study Group ${ }^{1}$
}

\begin{abstract}
ELF-EMF European Feasibility Study Group. Need for a European approach to the effects of extremely lowfrequency electromagnetic fields on cancer. Scand J Work Environ Health 1997;23(1):5-14.
\end{abstract}

\begin{abstract}
Background A European feasibility study on environmental exposure to extremely low-frequency electromagnetic fields (ELF-EMF) and cancer was conducted. The study was motivated by public health concern about possible adverse health effects associated with ELF-EMF exposure.

Methods A review of completed research in Europe was conducted. Information on the methods and accessibility of new epidemiologic studies were requested and reviewed.

Results Eight studies on environmental ELF-EMF exposure have been completed in Europe while 15 large studies are in progress. Although there is no known mechanism by which electric or magnetic fields of this frequency could play a role in the development of cancer or other adverse health effects, the results of the studies conducted so far provide some support for the hypothesis that they are associated with the incidence of childhood leukemia.

Conclusions and recommendations The best use of available data will be made through a pooled re-analysis of data, particularly those on childhood tumors. It is recommended to apply multiple methods for exposure assessment in view of the heterogeneity in the methods used in different studies. New multicenter case-referent studies should not be initiated until the results of the large on-going studies have been reported. Prospective cohort studies will have to be very large to identify moderate excess risks resulting from environmental exposure to ELF-EMF, and their feasibility should be discussed after the results of the on-going case-referent studies have been reported. A European collaborative approach will lead to greater statistical power and will assess the exposure-effect association under differing exposure patterns and distributions of potential confounding factors.
\end{abstract}

Key terms brain tumors, breast cancer, childhood cancer, electromagnetic fields, leukemia.

The transmission, distribution, and use of electric power gives rise to electric and magnetic fields in the extremely low-frequency band $(<300 \mathrm{hz}$ ) (ELF-EMF). Such fields are ubiquitous in modern society. High-voltage power lines are a very visible source of such fields, existing everywhere where there is electricity. There is no known mechanism by which electric or magnetic fields of this frequency, and of the strength that occurs in the general environment, could play a role in the development of cancer or other adverse health effects. However, epidemiologic research has indicated that there might be an association between residential and occupational exposure to magnetic fields and cancer.

A substantial number of committees and individual scientists has reviewed the scientific evidence related to the hypothesis of an association between exposure to

1 The group consists of: A Ahlbom, Karolinska Institute Stockholm, Sweden; F Alexander, University of Edinburgh Medical School, United Kingdom; G Draper, Childhood Cancer Research Group, Oxford, United Kingdom; E Casteleyn, VVIKMA, Belgium; J Clavel, INSERM U170, Villejuif, France; K Katsouyanni (coordinator), Department of Hygiene and Epidemiology, University of Athens, Medical School, Athens, Greece; E Petridou, Department of Hygiene and Epidemiology, University of Athens, Medical School, Athens, Greece; M Kogevinas, Institut Municipal d'Investigacio Medica, Barcelona, Spain; A McKinlay, National Radiological Protection Board, Oxford, United Kingdom; J Michaelis, University of Mainz, Germany; J Olsen, Danish Cancer Center, Copenhagen, Denmatk; E Roman, Leukaemia Research Fund, Leeds, Oxford, United Kingdom; A Salvan, National Research Council, Padova, Italy; T Tynes, Norwegian Radiation Protection Authorities, Norway; P Verkasalo, University of Helsinki, Helsinki, Finland.

Reprint requests to: Dr Klea Katsouyanni, Department of Hygiene and Epidemiology, University of Athens, Medical School, 75, Mikras Asias str, 11527 Athens (Goudi), Greece. 
ELF-EMF and cancer (1-4). These reviews have usually come to rather dismissing conclusions although with a varying degree of certainty. However, childhood leukemia is the area for which most reviewers believe the data provide strongest support for the hypothesis, and it is commonly suggested that it be investigated further. To date 11 published studies have addressed childhood leukemia and residential magnetic field exposure $(5-16)$. Despite small numbers and differences in study design and setting, the results appear to be rather consistent. If 3 studies with obvious design flaws are excluded, the remaining 7 studies have relative risk estimates in the range of up to $3.0(17-18)$. The reason for the cautious interpretation of the childhood data is 2 fold. First, the lack of a known biophysical mechanism results in fairly strong requirements on the epidemiologic evidence $(2,3)$. Second, there are certain issues even in the childhood leukemia studies that need to be addressed.

Within the framework of the Environment 91-94 Program a feasibility study on ELF-EMF and cancer in Europe was initiated. The purpose of this study was to (i) investigate the necessity and feasibility of a European epidemiologic study on the possible effects of ELF-EMF on childhood and adult cancers based on the status and results of current and previous projects; (ii) evaluate the advantages and disadvantages of several alternative or complementary epidemiologic approaches with which to study the possible effects of ELF-EMF on childhood and adult cancers; and (iii) provide suggestions for possible future investigations in this field. The present paper reports the results of this study, which was focused on nonoccupational exposure to ELF-EMF.

\section{Is there a need for further study?}

The evidence for a carcinogenic effect of ELF-EMF is limited, and there is no indication that large numbers of cases are attributable to this cause. There are, however, several reasons for suggesting that further studies should be carried out.

\section{Public interest}

The possible risks have received considerable publicity, and there is a good deal of interest and concern by the media and the public; people are raising questions about the possible risks of living close to electricity cables and overhead lines. It is not certain that such fears will be answered by the studies currently being carried out, especially when they deal mainly with exposures from domestic equipment.

\section{Individual risks}

Although it seems that the population attributable risk from exposure to transmission lines is small, it remains possible that people with high exposure levels might have high risks. This issue could be resolved by studies that include enough highly exposed people or by pooled analyses of existing and future studies that help estimate a dose-response relationship.

If there is in fact such a relationship, and if persons with high exposure can be identified, reducing exposures offers a possibility for prevention.

\section{Potentially indicative information on other risks or risks from other types of exposure}

Even if exposure to magnetic fields is not a causal factor increasing cancer risk, it may still be correlated with other exposures or other factors which are more relevant but have not yet been identified.

\section{Needs of electric power companies}

Because of public anxiety, power companies responsible for electricity transmission and distribution may meet opposition to some of their plans and may indeed face legal action. It is essential for them that the question of possible hazards from such activities be settled. If there is in fact no risk, they may be subjected to unnecessary restrictions and unjustified legal actions. If, on the other hand, there is a risk, they will need to take appropriate action concerning the location of their facilities.

\section{Is there a need for a European approach?}

Larger, or pooled studies will make it possible to estimate any effect more precisely and will give greater statistical power.

It is possible that similar levels of exposure, but arising from different sources, will be observed in different European countries. European-wide studies should make it possible to identify such differences and to determine whether the response is similar.

Confounding factors (ie, other possible risk factors statistically associated with EM exposure: socioeconomic status being a possible example) may vary between countries. European-wide studies may make it easier to rule out possible confounding effects (ie, risks apparently attributable to EM but actually attributable to these associated factors). In other words, an effect that persists in different circumstances or countries is less likely to be attributed to bias. Studies carried out by a collaborative European group will naturally require agreement and harmonization on the assessment of exposure. This is 
currently a problem in comparing results available from different studies.

\section{Electromagnetic fields - exposure assessment}

In previous epidemiologic studies a great variety of methods has been applied for EMF exposure assessment. This variety makes it difficult to summarize the published data and derive general conclusions. In the following text, we describe the different approaches. This description should serve as a basis for recommendations for future studies. New studies should aim at reproducing the findings of previous studies by using the same criteria for describing EMF exposure.

In most studies exposure assessment has been performed for the homes of the children and not for other sites, like their kindergartens or schools. Personal dosimetry has been applied in few instances. Exposure assessment of children's dwellings has been performed by describing the distance to EMF sources, by assigning wire codes, and by measuring or estimating the magnetic flux density.

Distances from the following EMF sources have been described: power lines (high voyage: $>200 \mathrm{kV}$, medium voltage $<200 \mathrm{kV}$ ), transformers, substations, radio transmission towers, railways and subways.

Wire codes are a classification scheme to characterize the wiring configuration of dwellings. The classification was first developed by Wertheimer \& Leeper, who introduced a 4-level wire code aimed at reflecting the amount of EMF exposure generated by an electrical power supply. This wire code was often collapsed into a 2-level wire code and also extended to a 5-level code (including the category "buried"). Recently, a simplified method for obtaining a comparable wire code has been described by Kaune \& Savitz (19).

Spot measurements have been performed at different locations in children's homes (eg, at the front door, in the bedroom, in the living room, etc). Measurements have been made under high-power and low-power conditions (ie, by either turning on or off available electrical appliances used in a household).

In addition to spot measurements, 24-hour measurements have also been performed. From these longer-term measurements the following parameters have been derived and used as exposure measures: mean value, median, 90th percentile, 95th percentile, difference between minimum and maximum, standard deviation of measurements, difference between certain quantiles of the measurement distribution. The following cut points have been used for assigning subjects to "highly exposed" groups: 0.01 to $0.8 \mu \mathrm{T}$.

In the most recent studies of exposure from power line estimates of magnetic flux density have been de- rived; it has been argued that these may provide a better indication of past exposure than the actual measurements. These estimations have been performed on the basis of different physical calculation models, taking into account distances from power lines, line configurations, height of pylons, and electrical load. They are not influenced by other local sources, for example, the electrical appliances used in households or the connection of an individual household to a power supply. Exposure estimates have been derived for the first year of life, for the year preceding the index diagnosis, for life-long exposure, and for the year with the maximal (average) exposures. The cut points for classification were chosen in a manner similar to that used for EMF measurements.

In an attempt to derive exposure measurements, children's dwellings have been chosen according to the address at the time of birth, the address at the time of diagnosis, and the address at the time of death. In addition, addresses of homes occupied for the longest time before the diagnosis and homes occupied just before the diagnosis have been investigated.

The heterogeneity of exposure criteria and study design has led to a variation in the fraction of exposed subjects in individual studies, the percentage of "highly exposed" persons ranging from $0.4 \%$ to $70 \%$ within the described study populations. Within the same studies the application of different exposure criteria has also led to different fractions of subjects being classified as "highly exposed". For example in the study of London et al (8), $10 \%$ of the subjects were exposed to a magnetic flux density of $>0.2 \mu \mathrm{T}$, while more than $50 \%$ were included in the "high-wire codes" category.

\section{Outcomes of interest}

Accurate data on the health outcome of interest and the exposure of interest are essential for good quality epidemiologic research. As a disease outcome, cancer is more amenable to study than many other adverse health events, partly because affected persons can be reliably identified in routinely compiled statistics, the three most common sources of cancer ascertainment being death certification, national cancer registration, and specialist tumor registration. For certain types of disease (eg, leukemia), the distribution of disease subtypes differs with respect to mortality and morbidity data.

As far as new studies of the relation between ELFEMF and cancer are concerned, incidence-based investigations are preferred. The prognoses for malignancies that have been linked with exposure to ELF-EMF (melanoma, female breast cancer, male breast cancer, brain cancer, and leukemia) continue to improve, the current European 5-year survival rates being greater 
than $50 \%$ for all but brain cancer. The rapid advances in the treatment of childhood leukemia are particularly noteworthy in this context, since 6 out of 10 children diagnosed with leukemia now survive into adulthood.

\section{Past and current research on extremely low- frequency electromagnetic field exposure and childhood cancer in Europe}

Table 1 summarizes the completed and published studies on ELF-EMF exposure and childhood cancer in Europe. Eight of them are from Scandinavia, the United Kingdom, or Greece.

Table 2 presents a summary of the on-going European research on which information was available. Fifteen studies are presented that are in progress in 8 different European countries, while 1 study is a multicountry coordinated European project. Some of these studies have been designed specifically to test the postulated associations between ELF-EMF exposure and cancer, while others have been initiated with the objective of investigating the etiology of certain neoplasms in the broader sense, including ELF-EMF exposure.
In addition to the aforementioned research activities, which is called a COST (coordination in science and technology) action, has been established within the framework of the European Commission. It concerns the biomedical effects of electromagnetic fields (20).

\section{Future research strategies}

\section{Use of available data or data under collection}

In general one should aim at obtaining as much information as possible out of already available data before collecting new data. There are several areas for which existing data can be put to use to obtain further insight.

One possibility would be to combine information from several different epidemiologic research centers across Europe. The objective would be to test the following three alternative hypotheses regarding explanations for the associations seen in epidemiologic research on EMF and childhood leukemia: (i) there is no association between EMF and childhood leukemia (ie, the observed associations are due to chance); (ii) an association between cancer risk and distance between homes and power lines exists that is independent of the magnetic field

Table 1. Past and current research on extremely low-frequency electromagnetic field (ELF-EMF) exposure and childhood cancer in Europe.

\begin{tabular}{|c|c|c|c|c|c|c|}
\hline Reference & Study design & Outcome & $\begin{array}{l}\text { ELF-EMF exposure } \\
\text { assessment }\end{array}$ & $\begin{array}{l}\text { Number of } \\
\text { subjects }\end{array}$ & $\begin{array}{l}\text { Number of } \\
\text { cases }\end{array}$ & $\begin{array}{l}\text { Number of } \\
\text { exposed cases }\end{array}$ \\
\hline $\begin{array}{l}\text { Tomenius, } 1986 \text { (12), } \\
\text { Stockholm }\end{array}$ & $\begin{array}{l}\text { Population based, case- } \\
\text { referent study, cases } \\
0-18 \text { years of age, } \\
1958-1973\end{array}$ & All neoplasms & $\begin{array}{l}\text { Spot measurements, } \\
\text { distance from power lines } \\
\text { (residence) }\end{array}$ & 1432 & 716 & $\begin{array}{l}<34 \\
(>0.3 \mu \mathrm{T})\end{array}$ \\
\hline $\begin{array}{l}\text { Myers et al, } 1990(9) \\
\text { Yorkshire }\end{array}$ & $\begin{array}{l}\text { Population based case- } \\
\text { referent study, cases } \\
0-15 \text { years of age, } \\
1970-1979\end{array}$ & All neoplasms & $\begin{array}{l}\text { Distance from power lines } \\
\text { (residence at birth), estimated } \\
\text { fields based on line-network } \\
\text { maps and load records }\end{array}$ & 962 & 374 & $\begin{array}{l}4 \% \\
(\geq 0.1 \mathrm{mG}) \\
10 \% \\
(<100 \mathrm{~m} \mathrm{dist})\end{array}$ \\
\hline $\begin{array}{l}\text { Coleman et al, } 1989 \\
\text { (5), SE England }\end{array}$ & $\begin{array}{l}\text { Hospital based case- } \\
\text { referent study, cases } \\
0-18 \text { years of age, } \\
1965-1980\end{array}$ & Leukemia & $\begin{array}{l}\text { Distance from lines or } \\
\text { substation (residence) }\end{array}$ & 225 & 84 & $\begin{array}{l}14 \\
(<50 \mathrm{~m} \text { from } \\
\text { substation) } \\
1 \\
(<100 \mathrm{~m} \text { from } \\
\text { line) }\end{array}$ \\
\hline $\begin{array}{l}\text { Verkasalo et al; } 1993 \\
\text { (13), Finland }\end{array}$ & $\begin{array}{l}\text { Retrospective cohort, children } \\
0-19 \text { years of age, } \\
\text { residence }<500 \mathrm{~m} \text { of } \\
\text { power lines, } 1974-1990\end{array}$ & $\begin{array}{l}\text { All neoplasms, } \\
\text { cVS tumors, } \\
\text { leukemia, } \\
\text { lymphoma }\end{array}$ & $\begin{array}{l}\text { Calculated fields, based on } \\
\text { data from typical locations } \\
\text { and distance }\end{array}$ & $\begin{array}{l}134 \\
800\end{array}$ & $\begin{array}{r}140 \\
39 \\
35 \\
15\end{array}$ & $\begin{array}{l}11 \\
(\geq 0.2 \mu \mathrm{T})\end{array}$ \\
\hline $\begin{array}{l}\text { Feyching \& Ahlbom, } \\
1993(6) \text {, Sweden }\end{array}$ & $\begin{array}{l}\text { Population-based case- } \\
\text { referent study, all subjects' } \\
\text { residences < } 300 \text { m from } \\
\text { power lines, } 1960-1985\end{array}$ & $\begin{array}{l}\text { All neoplasms, } \\
\text { leukemia, } \\
\text { brain tumors }\end{array}$ & $\begin{array}{l}\text { Spot measurements, distance } \\
\text { from lines, calculated fields } \\
\text { based on distance, line } \\
\text { configuration and load }\end{array}$ & 695 & 141 & $\stackrel{12}{(>0.2 \mu T)}$ \\
\hline $\begin{array}{l}\text { Olsen et al, } 1993(10) \text {, } \\
\text { Denmark }\end{array}$ & $\begin{array}{l}\text { Population based case- } \\
\text { referent study, cases } \\
0-15 \text { years of age, } \\
1968-1986\end{array}$ & $\begin{array}{l}\text { All neoplasms, } \\
\text { leukemia, } \\
\text { lymphoma, } \\
\text { CVS tumors }\end{array}$ & $\begin{array}{l}\text { Distance from lines (residence } \\
\text { before and after birth), } \\
\text { calculated fields based on } \\
\text { current loads, phase or density } \\
\text { of line and distance }\end{array}$ & 6495 & $\begin{array}{r}1707 \\
833 \\
250 \\
624\end{array}$ & $\begin{array}{l}10 \\
(\geq 0.1 \mu T)\end{array}$ \\
\hline $\begin{array}{l}\text { Petridou et al, } 1993 \\
\text { (16), Greece }\end{array}$ & $\begin{array}{l}\text { Hospital based case- } \\
\text { referent study, cases } \\
0-14 \text { years of age, } \\
1987-1991\end{array}$ & Leukemia & $\begin{array}{l}\text { Distance from lines and } \\
\text { substations }\end{array}$ & 410 & 170 & $\begin{array}{l}27 \\
(<5 \mathrm{~m} \text { from lines }) \\
100 \\
(<100 \mathrm{~m} \text { from } \\
\text { substation) }\end{array}$ \\
\hline
\end{tabular}

a Cutoff point for exposure definition in parentheses. 
Table 2. Ongoing studies in Europe on electromagnetic fields (ELF-EMF) and cancer.

\begin{tabular}{|c|c|c|c|c|c|c|c|}
\hline Organizationa & $\begin{array}{l}\text { Type of study/ } \\
\text { study base }\end{array}$ & Outcome & $\begin{array}{l}\text { ELF-EMF exposure } \\
\text { assessment }\end{array}$ & $\begin{array}{l}\text { Number of } \\
\text { subjects }\end{array}$ & $\begin{array}{l}\text { Number of } \\
\text { cases }\end{array}$ & $\begin{array}{l}\text { Number of } \\
\text { exposed cases }\end{array}$ & Time schedule \\
\hline $\begin{array}{l}\text { University of Edinburgh, } \\
\text { Medical School; } \\
\text { Freda Alexander, } \\
\text { European Concerted } \\
\text { Action: clustering of } \\
\text { childhood leukemia }\end{array}$ & $\begin{array}{l}\text { Identification of } \\
\text { small areas with } \\
\text { apparent leukemia } \\
\text { clusters and } \\
\text { comparison with } \\
\text { "control" areas }\end{array}$ & $\begin{array}{l}\text { Leukemia \& } \\
\text { subgroups }\end{array}$ & $\begin{array}{l}\text { Not yet completely } \\
\text { specified }\end{array}$ & $\begin{array}{l}\text { Data } \\
\text { assembled } \\
\text { from registries } \\
\text { in } 12 \\
\text { countries; } \\
\text { exact number } \\
\text { not yet known }\end{array}$ & Not yet known & Not yet known & Completion: 1996 \\
\hline $\begin{array}{l}\text { Department of Public } \\
\text { Health, University } \\
\text { of Helsinki }\end{array}$ & $\begin{array}{l}\text { Cohort of all Finnish } \\
\text { people with } \\
\text { residential } \\
\text { exposure to a } \\
\text { magnetic field of } \\
\geq 0.01 \mu T \text { at any } \\
\text { time in } 1970-1989\end{array}$ & $\begin{array}{l}\text { All types of } \\
\text { cancer } \\
\text { diagnosed in } \\
1974-1990\end{array}$ & $\begin{array}{l}\text { Computation of } \\
\text { residential } 50-\mathrm{Hz} \\
\text { magnetic fields } \\
\text { generated by } \\
\text { overhead power } \\
\text { lines; individual } \\
\text { estimates of } \\
\text { average annual } \\
\text { and cumulative } \\
\text { magnetic fields }\end{array}$ & $\begin{array}{l}3.5 \text { million } \\
\text { person-years }\end{array}$ & $\begin{array}{l}\text { Expected: } \\
\text { all types: } 8758 \text {; } \\
\text { female breast } \\
\text { cancer: } 1189 ; \\
\text { central nervous } \\
\text { system: } 45 ; \\
\text { melanoma: } 32 ; \\
\text { lung cancer: } 1213 \text {; } \\
\text { prostate cancer: } \\
\text { 559; leukemia: } 32\end{array}$ & $\begin{array}{l}\text { Expected: } \\
\text { with } 0.4 \mu \mathrm{T} \text {-years: } \\
\text { all: } 1227 ; \\
\text { female breast } \\
\text { cancer: } 164 ; \\
\text { CNS: } 45 ; \\
\text { melanoma: } 32 ; \\
\text { lung: } 174 ; \\
\text { prostate: } 183 \text {; } \\
\text { leukemia: } 32\end{array}$ & $\begin{array}{l}\text { Data collection } \\
1988-94 ; \\
\text { completion of } \\
\text { adult cancer study } \\
1996\end{array}$ \\
\hline $\begin{array}{l}\text { Administrative Unit } \\
\text { "Cote d' Or" (JP } \\
\text { Moutet, P Hillon) } \\
\text { France }\end{array}$ & $\begin{array}{l}\text { Exposure study on } \\
\text { a representative } \\
\text { sample of } 240 \\
\text { houses }\end{array}$ & .. & $\begin{array}{l}\text { 24-hour } \\
\text { measurements in } \\
\text { homes; wiring } \\
\text { configuration; } \\
\text { plan to develop } \\
\text { a French wiring } \\
\text { code }\end{array}$ & .. & " & .. & $\begin{array}{l}\text { Completion of the } \\
\text { study } 1996\end{array}$ \\
\hline $\begin{array}{l}\text { Belgian Ministry of } \\
\text { Science: Impulse } \\
\text { program "Health } \\
\text { Hazards: Effects of } \\
\text { EMF on Health" }\end{array}$ & $\begin{array}{l}\text { Controlled } \\
\text { experimental study } \\
\text { of human cells in } \\
\text { vitro }\end{array}$ & $\begin{array}{l}\text { Multiplication, } \\
\text { metabolic } \\
\text { activity, } \\
\text { response to } \\
\text { EGF, } \\
\text { contractile } \\
\text { properties, } \\
\text { attachment } \\
\text { to a collagen } \\
\text { substratum, } \\
\text { direct } \\
\text { imaging of } \\
\text { Cattious flux }\end{array}$ & $\begin{array}{l}\text { Cell cultures of } \\
\text { human origin } \\
\text { exposed to ELF- } \\
\text { EMF under strictly } \\
\text { controlled } \\
\text { conditions }\end{array}$ & .. & .. & .. & .. \\
\hline $\begin{array}{l}\text { Childhood Cancer } \\
\text { Research Group, } \\
\text { University of Oxford, } \\
\text { United Kingdom }\end{array}$ & $\begin{array}{l}\text { Case-control study } \\
\text { of childhood } \\
\text { leukemia and } \\
\text { other cancers } \\
\text { (below } 15 \text { years } \\
\text { of age); controls } \\
\text { matched by gender, } \\
\text { date and area of } \\
\text { birth }\end{array}$ & $\begin{array}{l}\text { Distance } \\
\text { from } \\
\text { overhead } \\
\text { lines; line- } \\
\text { based } \\
\text { exposure } \\
\text { estimates }\end{array}$ & $\begin{array}{l}\text { Distance from line } \\
\text { and historic data on } \\
\text { load and } \\
\text { characteristics of } \\
\text { line }\end{array}$ & $\begin{array}{l}\text { Leukemia } \\
\cong 10000 ; \\
\text { brain tumors } \\
\cong 7000 ; \\
\text { other cancers } \\
\cong 13000 ; \\
\text { equal number } \\
\text { of referents }\end{array}$ & $\begin{array}{l}\text { Same as number } \\
\text { of subjects }\end{array}$ & $\begin{array}{l}\text { Under the null } \\
\text { hypothesis: } \\
\text { leukemia } \cong 20- \\
30, \text { brain tumors } \\
15-20 \text { and other } \\
\text { cancers } 25-40 \\
\text { within } 100 \mathrm{~m} \\
\text { of the line, with } \\
\text { exposures of at } \\
\text { least } 0.1-0.2 \mu \mathrm{T} \\
\text { from this source }\end{array}$ & $\begin{array}{l}\text { Period of data } \\
\text { collection: } \\
\text { 1996-1998; } \\
\text { completion of the } \\
\text { study: March } 1999\end{array}$ \\
\hline $\begin{array}{l}\text { Institut für } \\
\text { Medizinische Statistik } \\
\text { und Dokumentation, } \\
\text { Johannes Gutenberg- } \\
\text { Universität Mainz, } \\
\text { Germany }\end{array}$ & $\begin{array}{l}\text { Nationwide } \\
\text { (former FRG) case- } \\
\text { referent study, } \\
\text { population-based }\end{array}$ & $\begin{array}{l}\text { Childhood } \\
\text { leukemia, } \\
\text { lymphoma, } \\
\text { other } \\
\text { selected } \\
\text { tumors }\end{array}$ & $\begin{array}{l}\text { Distance to power } \\
\text { lines; perhaps EMF } \\
\text { measurements }\end{array}$ & $\cong 3800$ & $\cong 1800$ cases & $\begin{array}{l}\text { Cutt-off points } \\
50 \text { and } 100 \mathrm{~m}\end{array}$ & $\begin{array}{l}\text { Completion of the } \\
\text { study: spring } 1997\end{array}$ \\
\hline $\begin{array}{l}\text { Institut für } \\
\text { Medizinische Statistik } \\
\text { und Dokumentation, } \\
\text { Johannes Gutenberg- } \\
\text { Universität Mainz, } \\
\text { Germany }\end{array}$ & $\begin{array}{l}\text { Case-referent } \\
\text { study; Lower- } \\
\text { Saxony, population- } \\
\text { based }\end{array}$ & $\begin{array}{l}\text { Childhood } \\
\text { leukemia, } \\
\text { other } \\
\text { selected } \\
\text { tumors }\end{array}$ & $\begin{array}{l}\text { Distance to power } \\
\text { lines; spot } \\
\text { measurements; } \\
\text { 24-hour } \\
\text { measurements; } \\
\text { (wire codes for a } \\
\text { subgroup only) }\end{array}$ & 770 & $\begin{array}{l}160 \text { leukemia, } \\
170 \text { other tumors, } \\
440 \text { referents }\end{array}$ & $\begin{array}{l}\text { Cut-off points } \\
0.1 \mu \mathrm{T} \text { and } 0.2 \mu \mathrm{T} \\
\text { and } 50 \text { and } 100 \mathrm{~m}\end{array}$ & $\begin{array}{l}\text { Period of data } \\
\text { collection: } \\
\text { interviews from } \\
\text { 1992-1995; } \\
\text { completion of the } \\
\text { study: end of } 1995\end{array}$ \\
\hline $\begin{array}{l}\text { Cancer Registry of } \\
\text { Norway/NRPA } \\
\text { (Tore Tynes) }\end{array}$ & $\begin{array}{l}\text { Nested case-referent } \\
\text { study; breast cancer } \\
\text { in women living } \\
\text { close to overhead } \\
\text { power lines }\end{array}$ & $\begin{array}{l}\text { Female } \\
\text { breast } \\
\text { cancer }\end{array}$ & $\begin{array}{l}\text { Calculated magnetic } \\
\text { and electric fields } \\
\text { based on power- } \\
\text { line data }\end{array}$ & $\begin{array}{l}500 \text { cases and } \\
1000 \text { referents }\end{array}$ & $\begin{array}{l}\text { Breast cancer } \\
\text { (500 cases) }\end{array}$ & $\begin{array}{l}50 \text { cases? (cut-off } \\
0.05 \mu T \text { ) }\end{array}$ & $\begin{array}{l}\text { Period of data } \\
\text { collection: } \\
1989 \text { to } 1995 ; \\
\text { completion of the } \\
\text { study: } 1996\end{array}$ \\
\hline $\begin{array}{l}\text { University of Oslo, } \\
\text { Norway/Physics } \\
\text { department (Arnt } \\
\text { Inge Vistnes) }\end{array}$ & $\begin{array}{l}\text { Magnetic field } \\
\text { dosimetry study on } \\
\text { children living close } \\
\text { to overhead power } \\
\text { lines }\end{array}$ & .. & $\begin{array}{l}\text { 24-hour dosimeter } \\
\text { measurements; } \\
\text { calculated magnetic } \\
\text { fields based on } \\
\text { power-line data }\end{array}$ & $\begin{array}{l}65 \text { school- } \\
\text { children }\end{array}$ & .. & .. & $\begin{array}{l}\text { Period of data } \\
\text { collection: } 1989 \text { to } \\
\text { 1995; completion of } \\
\text { the study: } 1995\end{array}$ \\
\hline $\begin{array}{l}\text { Cancer Registry of } \\
\text { Norway/NRPA } \\
\text { (Tore Tynes) }\end{array}$ & $\begin{array}{l}\text { Nested case-referent } \\
\text { study; childhood } \\
\text { cancer in children } \\
\text { living close to } \\
\text { overhead power } \\
\text { lines }\end{array}$ & $\begin{array}{l}\text { Childhood } \\
\text { cancer }\end{array}$ & $\begin{array}{l}\text { Calculated magnetic } \\
\text { and electric fields } \\
\text { based on power- } \\
\text { line data }\end{array}$ & $\begin{array}{l}500 \text { cases and } \\
2004 \text { referents }\end{array}$ & $\begin{array}{l}\text { All cancers } \\
\text { (500 cases) }\end{array}$ & $\begin{array}{l}46 \text { cases } \\
\text { (cut-off } 0.05 \mu \mathrm{T} \text { ) }\end{array}$ & $\begin{array}{l}\text { Period of data } \\
\text { collection: } 1989 \text { to } \\
\text { 1995; completion of } \\
\text { the study: } 1995\end{array}$ \\
\hline
\end{tabular}


Table 2. Continued

\begin{tabular}{|c|c|c|c|c|c|c|c|}
\hline Organization ${ }^{2}$ & $\begin{array}{l}\text { Type of study/ } \\
\text { study base }\end{array}$ & Outcome & $\begin{array}{l}\text { ELF-EMF exposure } \\
\text { assessment }\end{array}$ & $\begin{array}{l}\text { Number of } \\
\text { subjects }\end{array}$ & $\begin{array}{l}\text { Number of } \\
\text { cases }\end{array}$ & $\begin{array}{l}\text { Number of } \\
\text { exposed cases }\end{array}$ & Time schedule \\
\hline $\begin{array}{l}\text { Multicenter study } \\
\text { covering all of Great } \\
\text { Britain }\end{array}$ & $\begin{array}{l}\text { Case-referent } \\
\text { study }\end{array}$ & $\begin{array}{l}\text { All cancers } \\
\text { diagnosed } \\
<15 \text { years }\end{array}$ & $\begin{array}{l}\text { EMF } \\
\text { measurements } \\
\text { (spot and } \\
\text { continuous), data } \\
\text { from electricity } \\
\text { companies, } \\
\text { questionnaire } \\
\text { data on electric } \\
\text { appliance usage }\end{array}$ & $\begin{array}{l}\text { Approximately } \\
6000 \text { (3000 } \\
\text { cases and } \\
3000 \\
\text { referents) }\end{array}$ & $\begin{array}{l}\text { Approximately } \\
1000 \text { acute } \\
\text { lymphoblastic } \\
\text { leukemia (ALLs) } \\
+2000 \text { other } \\
\text { childhood } \\
\text { cancers }\end{array}$ & $\begin{array}{l}\text { Approximately } \\
300 \text { exposed to } \\
150 \text { nT or more } \\
\text { (assuming } \\
\text { relative risk }=1.0 \text { ) } \\
\end{array}$ & $\begin{array}{l}\text { Period of data } \\
\text { collection: incident } \\
\text { cases 1992-1995 } \\
\text { for England and } \\
\text { Wales and } 1991- \\
1994 \text { for Scotland; } \\
\text { completion of } \\
\text { the study } 1997\end{array}$ \\
\hline $\begin{array}{l}\text { Cancer Epidemiology } \\
\text { Unit, Childhood } \\
\text { Cancer Registry, } \\
\text { Torino, Italy }\end{array}$ & $\begin{array}{l}\text { Case-referent- } \\
\text { pilot study will } \\
\text { start in } 1996\end{array}$ & $\begin{array}{l}\text { Leukemia in } \\
\text { children }\end{array}$ & $\begin{array}{l}\text { Expert assessment, } \\
\text { questionnaire } \\
\text { for exposure to } \\
\text { domestic electrical } \\
\text { machines; } \\
\text { the feasibility of } \\
\text { field measures } \\
\text { to be tested }\end{array}$ & $\begin{array}{l}\text { Expected } 200 \\
\text { cases per } \\
\text { year }\end{array}$ & $\begin{array}{l}200 \text { cases per } \\
\text { year }\end{array}$ & Not yet known & $\begin{array}{l}\text { Pilot studiy starting } \\
\text { in January 1996; } \\
\text { data collection } \\
\text { starting in January } \\
\text { 1997; completion of } \\
\text { the study end of } \\
1998 \text { or mid-1999 }\end{array}$ \\
\hline $\begin{array}{l}\text { Department of Hygiene } \\
\text { and Epidemiology, } \\
\text { Athens University } \\
\text { Medical School }\end{array}$ & $\begin{array}{l}\text { Case-referent } \\
\text { study in Attica and } \\
\text { Grete, Greece, with } \\
\text { outpatient hospital } \\
\text { referents }\end{array}$ & $\begin{array}{l}\text { Childhood } \\
\text { leukemia }\end{array}$ & $\begin{array}{l}\text { Distance of } \\
\text { residence from } \\
\text { (a) electricity } \\
\text { substations and } \\
\text { (b) visible power } \\
\text { lines }\end{array}$ & $\begin{array}{l}136 \text { cases } \\
\text { and } 187 \\
\text { referents }\end{array}$ & $\begin{array}{l}\text { See number of } \\
\text { subjects }\end{array}$ & $\begin{array}{l}\text { a) Distance from } \\
\text { electricity } \\
\text { substation } \\
(<100 \mathrm{~m}): 5 \text { cases } \\
\text { and } 13 \text { referents; } \\
\text { b) distance from } \\
\text { power lines }(<5 \mathrm{~m}) \text { : } \\
27 \text { cases and } \\
33 \text { referents; } \\
\text { ( } 5 \text { - } 49 \mathrm{~m}) \text { : } \\
69 \text { cases and } \\
99 \text { referents }\end{array}$ & $\begin{array}{l}\text { Completion of the } \\
\text { study } 1996\end{array}$ \\
\hline $\begin{array}{l}\text { Department of Hygiene } \\
\text { and Epidemiology, } \\
\text { Athens University } \\
\text { Medical School }\end{array}$ & $\begin{array}{l}\text { Case-referent } \\
\text { study }\end{array}$ & $\begin{array}{l}\text { Childhood } \\
\text { leukemia }\end{array}$ & $\begin{array}{l}\text { Wire code } \\
\text { configuration } \\
\text { estimates, spot } \\
\text { measurements }\end{array}$ & $\begin{array}{l}160 \text { incidence } \\
\text { cases of } \\
\text { childhood } \\
\text { leukemia } \\
320 \text { hospital } \\
\text { referents }\end{array}$ & $\begin{array}{l}\text { See number of } \\
\text { subjects }\end{array}$ & Not yet known & $\begin{array}{l}\text { Period of data } \\
\text { collection } 1993- \\
\text { 1995; completion } \\
\text { of the study } \\
\text { 30 June } 1996\end{array}$ \\
\hline $\begin{array}{l}\text { Several centers in } \\
\text { France (INSERM- } \\
\text { U170 - J Clavel) }\end{array}$ & $\begin{array}{l}\text { Hospital-based } \\
\text { case-referent study }\end{array}$ & $\begin{array}{l}\text { All incident } \\
\text { leukemias and } \\
\text { lymphomas }\end{array}$ & $\begin{array}{l}\text { Distance to high- } \\
\text { voltage power } \\
\text { lines: question- } \\
\text { naire on some } \\
\text { domestic electrical } \\
\text { appliance; EMF } \\
\text { measurements } \\
\text { (lifelong residential } \\
\text { history recorded) }\end{array}$ & $\begin{array}{l}300 \text { cases and } \\
300 \text { referents }\end{array}$ & $\begin{array}{l}\text { See number of } \\
\text { subjects }\end{array}$ & Not yet known & $\begin{array}{l}\text { Completion of the } \\
\text { study end of } 1997\end{array}$ \\
\hline
\end{tabular}

a Organization in change of research.

generated by the power line; (iii) the associations between magnetic fields and cancer can be explained by confounding from some other specified putative risk factor, such as pesticide or car exhaust. By combining information from centers across Europe, two essential objectives would be achieved. First, the number of cases with elevated residential magnetic field exposure would be sufficiently large to allow for the required analyses, Second, since countries from all over Europe would be included, an appropriate variation in exposure conditions would be available to help discriminate between the different possible explanations for the observed associations.

Data could also be used to coordinate, as far as possible, analyses from parallel studies. Such an approach was taken, to some extent, with three Nordic studies that have already been published $(6,10,13)$. Such coordination makes comparisons more feasible and adds to the numbers behind any particular analysis. Much of the epidemiologic research on magnetic fields and cancer has been undertaken in North America, in particular in the United States. Thus it would also make sense to consider collaboration with groups outside Europe if a combined analysis were indeed planned.

During the project there was some discussion about whether the relation between other adverse health outcomes and ELF-EMF should be investigated in existing data sets. Although associations have been suggested for conditions such as miscarriage, suicide, depression, and Alzheimer's disease, it was felt that combined analyses were not warranted at present.

Combining data from several studies might prove particularly useful in evaluations of various exposure assessment methods, especially since electricity distribution and transmission systems are set up differently across countries in Europe. In addition such information 
is crucial since most reviewers have stressed the difficulties involved in exposure assessment and the need to advance the understanding of what aspect of magnetic fields is the most related to cancer causation.

\section{New multicenter studies}

The existing evidence for an association between cancer and exposure to ELF-EMF is far from conclusive. Increased risks have been suggested for 5 cancer sites, namely, leukemia, brain cancer, female breast cancer, male breast cancer, and melanoma. For childhood leukemia - where the epidemiologic data are perhaps the most persuasive - more detailed information on the nature of exposure and the diagnostic subtypes involved is required before meaningful conclusions can be drawn. For other childhood and adult malignancies - for which the existing epidemiologic data are even more sparse and contradictory - more precise information on larger numbers of exposed persons are needed to confirm or refute previously reported associations.

As can be seen from table 2, the possible carcinogenic effects of ELF-EMF are being investigated in several ongoing European studies. Given the size and scope of the ongoing childhood cancer investigations, it would seem prudent to wait until their findings are available before initiating further research involving large amounts of data collection. The information from these extensive investigations should make a significant contribution to the accumulating body of knowledge about the postulated association between ELF-EMF and childhood cancer, particularly leukemia. In this context, a more important issue to be addressed is the feasibility of formally pooling similar data collected by different European centers.

For nonhematological malignancies, fewer studies are currently underway. In the event of new studies, a coordinated approach aimed at ensuring the collection of good quality exposure data is clearly desirable. Furthermore, researchers beginning new etiologic investigations on malignancies for which ELF-EMF have been suggested as a risk factor could perhaps be actively encouraged to incorporate ELF-EMF exposure assessments into their protocol.

\section{Assessing exposure to ELF-EMF in areas with identified leukemia clusters}

In the ongoing collaborative study funded by the Biomed I Programme of the European Union (table 2), clusters of childhood leukemia are being identified and examined in Europe. The objectives of the study are in part to relate clusters and the intensity of clustering to community occupational or environmental exposure to known or suspected leukemogens. One such suspected leukemogen is ELF-EMF.

Leukemia cluster areas and comparison areas will be identified through population-based cancer registers in
Europe, and a set of questions will be constructed for each of the selected areas. The questions will be answered by local researchers who are blind to the cluster or comparison status of each area. The questions on ELF-EMF will include the number and total length of overhead power lines of $\geq 100 \mathrm{kV}$ passing through the area, the relative size of the population living within a certain, predefined distance from the power lines, the number of electrical power stations, and number of large transformer stations within the area. Other characteristics will also be retrieved.

\section{Exposure study}

Most epidemiologic studies related to the putative health effects of exposure to electromagnetic fields have used surrogate measures of personal exposure. The surrogate measures have included wire codes (based on the density and type of wiring adjacent to homes), spot measurements of magnetic flux density outside and inside homes, and the proximity of external sources such as power lines and occupational matrices.

The recent availability of instruments capable of recording personal exposure to magnetic fields as a function of time and integrated over time now provides the opportunity to estimate personal exposure through a combination of spot and more prolonged environmental measurements, validated as adequate surrogates of personal exposure. With respect to exposure from power lines, with the co-operation of the relevant power utility, it may be possible to correct exposure data through a comparison with historical data on the variations of electrical power loads on the lines.

Given the resources available, any protocol involving the compilation of measurements and other behavioral data will be a compromise. In addition, since convincing biological data are lacking as to the mechanism for carcinogenesis from electromagnetic field exposure, the exposure metric is not obvious. It does not seem unreasonable to assume that risk might in some way be proportional to time-integrated exposure, that is, to time-weighted exposure. However, the possibility cannot be ruled out that the appropriate measure of dose is peak transient exposures.

An epidemiologic program involving the measurement of electromagnetic fields requires adequate training of the operators in relation to the correct use and checking of the instruments and adequate physics and computing support in respect to the checking, calibration and maintenance of the instruments and the downloading and handling of the large amounts of temporal data generated.

\section{Prospective cohort studies}

In prospective cohort studies the cohort is assembled and characterized according to its exposure to ELF-EMF, the 
subjects are followed over a period of time, and the disease occurrence or mortality is registered. The main advantage of this type of study, as opposed to retrospective cohort and case-referent studies, is that exposure to ELF-EMF is measured before disease occurrence on one or more separate occasions. Other risk factors (potential confounders), such as smoking and socioeconomic status, traffic density, and effect modifiers, can be determined concurrently. Such a study helps to overcome arguments concerning the reliance of case-referent studies on exposure modeling rather than on direct measurements of ELF-EMF. A possible design would include spot or, preferably, more-extended measurements at home, school, nurseries, and other identified places at the time of cohort recruitment and during subsequent time periods. Use of stochastic exposure models would probably be necessary to allocate exposure estimates to subjects with no or limited exposure data.

Given the rarity of the outcomes of interest (childhood cancers) and the expected low risk associated with residential exposure to ELF-EMF, a prospective cohort study would have to be very large to have enough statistical power to detect any excess risk. This need constitutes the principal disadvantage (nearly prohibiting factor) of prospective study designs for studies involving

Table 3. All childhood cancers - Number of subjects that should be included in a prospective study of children aged $0-10$ years and followed for 5 years in order to identify a statistically significant (alpha $=0.05$ ) result with a power of $80 \%$. Estimates are provided for different magnitudes of risk and for two sampling fractions between the exposed and unexposed persons. A cumulative incidence of 1800 per million has been assumed for the ages of $0-14$ years.

\begin{tabular}{lcc}
\hline Relative risk & \multicolumn{2}{c}{ Total number of subjects } \\
\cline { 2 - 3 } & $\begin{array}{c}\text { Ratio of exposed to } \\
\text { unexposed }(1: 1)\end{array}$ & $\begin{array}{c}\text { Ratio of exposed to } \\
\text { unexposed }(1: 9)\end{array}$ \\
\hline 1.2 & 1471154 & 3966180 \\
1.5 & 274602 & 712470 \\
2.0 & 84952 & 209450 \\
\hline
\end{tabular}

Table 4. All childhood leukemia - Number of subjects that should be included in a prospective study of children aged $0-10$ years and followed for 5 years in order to identify a statistically significant (alpha $=0.05$ ) result with a power of $80 \%$. Estimates are provided for different magnitudes of risk and for two sampling fractions between the exposed and unexposed persons. A cumulative incidence of 600 per million has been assumed for the ages of $0-14$ years.

\begin{tabular}{lcc}
\hline Relative risk & \multicolumn{2}{c}{ Total number of subjects } \\
\cline { 2 - 3 } & $\begin{array}{c}\text { Ratio of exposed to } \\
\text { unexposed }(1: 1)\end{array}$ & $\begin{array}{c}\text { Ratio of exposed to } \\
\text { unexposed }(1: 9)\end{array}$ \\
\hline 1.2 & 4415366 & 11903540 \\
1.5 & 824204 & 2138370 \\
2.0 & 255002 & 628660 \\
\hline
\end{tabular}

children. Table 3 (for all childhood cancers) and table 4 (for leukemia) present estimates of the necessary size of a study for increases in risk of the order of $20 \%, 50 \%$ and $100 \%$. The cumulative incidences (per year) of all childhood cancers and leukemia have been derived from Parkin et al (21). It was assumed that, in western Europe, the cumulative incidence for all childhood cancers (ie, those occurring in the first 15 years of life) is around 1800 per million and for leukemias around 600 per million. It was also assumed that the cohort includes children from 0 to 10 years of age, who will then be followed for 5 years. Estimates are provided for a study including an equal proportion of exposed and unexposed persons (ratio 1:1), as well as for studies including a much higher proportion of unexposed persons (1:9 ratio). The latter scenario seems more realistic since only about $10 \%$ or less of the nonworking population would have average exposures above $0.1-0.2 \mu \mathrm{T}$. The estimates of the prevalence of exposure are based on the few existing data from selected countries, and they may prove to be inaccurate. The costs and complexity of a prospective cohort design indicates that, for the time being, it is more reasonable to postpone studies focusing on childhood cancer until all case-referent studies have been completed. Once the findings of the case-referent studies are available, recommendations can be made on how to continue.

\section{Retrospective cohort studies}

With retrospective cohort studies, the study population and the exposure of subjects during past time periods are defined according to existing records. Follow-up takes place retrospectively, and in some studies it also incorporates a prospective approach. An example of a retrospective cohort study would be an investigation focusing on all children having attended schools with high ELFEMF exposures.

This design does not respond to issues concerning the validity of exposure assessment since the ELF-EMF exposure must be reconstructed a posteriori on the basis of existing data, such as electricity consumption bills or questionnaires or distance measures, with or without the use of exposure modeling techniques. These designs may, however, prove useful in addressing important public health issues that have been raised concerning exposure to ELF-EMF, such as the risks of attending schools built close to or under power lines. Cohorts of schoolchildren could be reconstructed on the basis of school records and followed retrospectively. Children having attended or attending schools built in other locations of similar socioeconomic and educational characteristics could be used as a comparison. Provided the study is large enough and the classification of exposure at school is adequate (even if retrospective), such studies could provide relatively low-cost guidelines for risk assessment and public health action concerning this source of 
ELF-EMF exposure. It should be noted, however, that this type of study would incorporate a considerable amount of misclassification since exposure differs considerably within schools and also because other sources of exposure (potentially more important) would not be taken into account. Another example of a retrospective cohort study identifying a special exposure circumstance is a study on children put in incubators after birth. In such a study, exposure evaluation would be more straightforward (concerning at least the first period of life), since babies in incubators would clearly be highly exposed to ELF-EMF. This type of study would have problems similar to those of other retrospective studies in reconstructing exposure in other time periods and other locations.

Thus it seems that retrospective cohort studies do not provide an advantage relative to case-referent studies concerning the assessment of exposure. Their conduct could be recommended in specific circumstances as a relatively cheap design focusing on specific "high" risk populations.

\section{Other related exposures}

Public exposure to radio-frequency field and microwave radiation is rapidly increasing (cellular phones). In many European countries, at present, 1 out of 4 adult inhabitants are users of cellular phones. Cohorts of cellular phone users could therefore be established at the European level. Nested case-referent cancer studies within these cohorts would be of interest (brain tumors, leukemia and female breast cancer). Telephone bills could be used as possible exposure surrogates.

A European cancer study on people living close to cellular towers and television and radio antennas would perhaps also be of interest. Studies at the national level are too small to provide sufficient numbers of exposed cases. A multicenter European study would have the statistical power to evaluate a possible effect related to such exposure.

Cancer studies of users of visual display terminals (VDT) may also be of interest at the European level. These devices have been used since 1970, and their usage is now widespread. Cohorts could be established and nested case-referent studies of selected cancer sites in cohorts of male and female VDT users could, in theory, be performed.

\section{Recommendations}

In view of the heterogeneity of existing exposure assessment methodologies, it is recommended that multiple methods be applied where possible. There would be less controversy with regard to the relevant outcomes, and it is suggested that, for certain malignancies, further inves- tigation is needed. A review of completed and ongoing research in Europe illustrates the need for coordination. Several alternative approaches to further research on this issue have been considered.

It is recommended (i) that the best use of available data be made through reanalysis, pooled analysis, metaanalysis and the like, (ii) that new multicenter studies should not be initiated until the results of large ongoing studies have been reported, (iii) that ELF-EMF exposure should be calculated for areas with identified leukemia clusters and reference areas within the framework of the ongoing European multicenter study, (iv) that consideration should be given to pooling existing European data on personal dosimetry since data are expensive to collect. New investigations on exposure should, perhaps, be initiated because of the paucity of good exposure data, (v) that the possibility of organizing a cohort study should be discussed after the results of on-going studies have been reported.

\section{Acknowledgments}

This study was supported by the EC Environment 9194 Programme, contract EV5V-CT94-0562. The scientist responsible in the EC was Dr Nolan.

\section{References}

1. Ahlbom A. A review of the epidemiologic literature on magnetic fields and cancer. Scand J Work Environ Health 1988;14: $337-43$.

2. National Radiological Protection Board (NRPB). Report of an advisory group on non-ionising radiation: electromagnetic fields and the risk of cancer. London: NRPB, 1992. Documents of NRPB, vol 3 .

3. Oak Ridge Associated Universities. Health effects of low frequency electric and magnetic fields. Oak Ridge (TN): Oak Ridge Associated Universities, 1992. ORAU92F8.

4. Savitz DA, Ahlbom A. Epidemiologic evidence on cancer in relation to residential and occupational exposures. In: Carpenter DO, Ayrapetyan S, editors. Biologic-effects of electric and magnetic fields; vol 2. New York (NY): Academic Press, 1994:233-62

5. Coleman M, Bell C, Taylor H, Primic-Zakelj M. Leukaemia and residence near electricity transmission equipment: a casecontrol study. Br J Cancer 1989;60:793-98.

6. Feychting M, Ahlbom A. Magnetic fields and cancer in children residing near Swedish high voltage power lines. Am J Epidemiol 1993;138:467-81.

7. Fulton JP, Cobb S, Preble L, Leone L, Forman E. Electrical wiring configurations and childhood leukaemia in Rhode Island. Am J Epidemiol 1980;111:292 - 6.

8. London S, Thomas D, Bowman J, Sobel E, Peters J. Exposure to residential electric and magnetic fields and risk of childhood leukaemia. Am J Epidemiol 1991;134:923-7.

9. Myers A, Clayden A, Cartwright R, Cartwright S. Childhood 
cancer and overhead powerlines: a case control study. Br J Cancer 1990;62:1008-14.

10. Olsen JH, Nielsen A, Schulgen G. Residence near high-voltage facilities and the risk of cancer in children. BMJ 1993;307: $891-5$.

11. Savitz D, Wachtel H, Barnes FA, John EM. Case-control study of childhood cancer and exposure to 60-hertz electric and magnetic fields. Am J Epidemiol 1988;128:21 - 38.

12. Tomenius L. 50-Hz electromagnetic environment and the incidence of childhood tumors in Stockholm county. Bioelectromagnetics 1986;7:191 - 207.

13. Verkasalo PK, Pukkala E, Hongisto MY, Valijus JE, Jarvinen PJ, Heikkila KV, et al. Risk of cancer in Finnish children living close to power lines. BMJ 1993;895-9.

14. Wertheimer $N$, Leeper E. Electrical wiring configurations and childhood cancer. Am J Epidemiol 1979;109:273-84.

15. Fajardo-Gutierrez A, Garduno-Espinosa J, Yamamoto-Kimura L, et al. Residence close to high-tension electric power lines and its association with leukemia in children [in Spanish]. Boletin Medico del Hospital Infantil de Mexico 1993;50:328. English abstract.
16. Petridou E, Kassimos D, Kalmant M, Kosmidis H, Haidas S, Flytzani $\mathrm{V}$, et al. Age of exposure to infections and risk of childhood leukemia. BMJ 1993;307:774 -5.

17. Ahlbom A, Feychting M. EMF and cancer [letter]. Science 1993;260:14-6.

18. Feychting M, Ahlbom A. Meta analysis of epidemiological studies - a critical reflection with special reference to magnetic fields and childhood cancer. Cancer Causes Control. In press.

19. Kaune WT, Savitz DA. Simplification of the Wertheimer and Leeper wire code. Bioelectromagnetics 1994;15:275-82.

20. Koren Z. Activity report leading to COST 244 formation. Zagreb: University of Zagreb, Faculty of electrical engineering. Reference $244 \mathrm{TD}(92) 001$.

21. Parkin DM, Stiller CA, Draper GJ, Bieber CA, Terracini B, Young JL, editors. International incidence of childhood cancer. Lyon: International Agency for Research on Cancer, 1988. Scientific publications, no 87 .

Received for publication: 2 July 1996 